\title{
Reachable Set Analysis for Singular Systems
}

\author{
Zhiguang Feng ${ }^{1}$, James Lam ${ }^{2}$ \\ 1. College of Information Science and Technology, Bohai University, Jinzhou, 121013, P. R. China \\ E-mail: congdian@gmail.com \\ 2. Department of Mechanical Engineering, The University of Hong Kong, Pokfulam Road, Hong Kong \\ E-mail: james.lam@hku.hk
}

\begin{abstract}
In this paper, the problem of reachable sets estimation of singular systems is investigated. Based on the Lyapunov method, a sufficient condition is established in terms of linear matrix inequality (LMI) to guarantee that the reachable set of a singular system is bounded by a ball. The effectiveness of the obtained results in this paper is illustrated by numerical examples.
\end{abstract}

Key Words: Linear Matrix Inequalities, Reachable Sets, Singular Systems

\section{Introduction}

Reachable set estimation of dynamic systems is to derive some closed bounded set to bound the set of all the states from the origin by inputs with bounded peak value, which is an important problem in the robust control theory [5], [7], [26] and has attracted the attention of many researchers. To mention a few, by using the S-procedure, the problem is investigated in [1] with the result derived in terms of liner matrix inequality (LMI) for the linear systems without time-delay. For the case with time-delay, the problem is first solved in [5] based on Lyapunov-Razumikhin method. An improvement result is proposed in [8] by using the modified Lyapunov-Krasovskii type functional. By utilizing convex-full properties in [10] and constructing the maximal Lyapunov-Krasovskii functional in [26], respectively, both results further improve that in [8]. Very recently, the authors in [13] presented an improved bound of the reachable set using the delay partitioning method. However, up to now, a unique result of other systems is obtained in [16] which investigates the reachable sets for linear neutral systems with disturbances.

In this paper, we obtain a ball that bounds the reachable set of singular system for the first time. Singular systems also called descriptor systems, differential-algebraic equation systems, generalized state-space systems, can better describe the behavior of some physical system than state-space ones [4], [22], [23]. Singular systems have been widely found in many practical systems, such as chemical processes [9], circuit systems [14], economic systems [12] and aircraft modeling [17]. Apart from their practical significance, they are of theoretical importance and have received a great deal of attention in recent years owing to their fundamental differences from state-space systems. Many fundamental concepts and results based on the theory of state-space systems have been successfully extended to singular systems, see stability and stabilization [21], [25], $H_{\infty}$ control [3], [18], [24], model reduction [11], [20], and dissipativity analysis [2], [19].

In this paper, we extend the reachable set estimation result to singular system for the first time. By using the Lyapunov-Krasovskii method, a sufficient condition is proposed in terms of LMI and a ball is obtained to bound all

This work was partially supported by GRF HKU 7137/13E, by Liaoning Provincial Natural Science Foundation of China (2013020227) and by the National Natural Science Foundation of China (61304063). states set of singular systems starting from the origin with a bounded input. Finally, numerical examples are given to illustrate the effectiveness of the proposed results.

The rest of this paper is briefly outlined as follows. In Section 2, the reachable set estimation problem of singular systems is formulated and solved. Two illustrative examples are provided in Section 3 to show the effectiveness of our results. We conclude the paper in Section 4.

Notation: The notation used throughout the paper is standard. $\mathbb{R}^{n}$ denotes the $n$-dimensional Euclidean space and $P>0(\geq 0)$ means that $P$ is real symmetric and positive definite (semi-definite); $I$ and 0 refer to the identity matrix and zero matrix with compatible dimensions; $\star$ stands for the symmetric terms in a symmetric matrix and $\operatorname{sym}(A)$ is defined as $A+A^{T} ; \bullet$ represents matrices that are not relevant with our discussion; $(M)_{m \times m}$ is the matrix composed of elements of first $m$ rows and $m$ columns of matrix $M ;\|\cdot\|$ refers to the Euclidean vector norm. Matrices are assumed to be compatible for algebraic operations if their dimensions are not explicitly stated.

\section{Reachable Sets of Singular System}

Consider a class of linear continuous-time singular systems described by

$$
\left\{\begin{aligned}
E \dot{x}(t) & =A x(t)+B w(t) \\
x(0) & \equiv 0
\end{aligned}\right.
$$

where $x(t) \in \mathbb{R}^{n}$ is the state vector; matrices $E, A$ and $B$ are constant matrices with appropriate dimensions and $\operatorname{rank}(E)=n_{1} ; w(t) \in \mathbb{R}^{l}$ represents a disturbance which satisfies

$$
w^{T}(t) w(t) \leq \bar{w}^{2}
$$

Before moving on, we give some definitions and lemmas which will be used in deriving the main results.

\section{Definition 1 [22]}

1) The singular system in (1) is said to be regular if $\operatorname{det}(s E-A)$ is not identically zero.

2) The singular system in (1) is said to be impulse free if $\operatorname{deg}\{\operatorname{det}(s E-A)\}=\operatorname{rank} E$.

Lemma 1 [22] If system (1) is regular and impulse free, 
there exist two non-singular matrices $M$ and $N$ such that

$$
M E N=\left[\begin{array}{ll}
I & 0 \\
0 & 0
\end{array}\right], M A N=\left[\begin{array}{cc}
A_{1} & 0 \\
0 & I
\end{array}\right]
$$

Let $\tilde{x}(t)=N^{-1} x(t)=\left[\begin{array}{c}\tilde{x}_{1}(t) \\ \tilde{x}_{2}(t)\end{array}\right]$, where $\tilde{x}_{1}(t) \in \mathbb{R}^{n_{1}}$ and $\tilde{x}_{2}(t) \in$ $\mathbb{R}^{n-n_{1}}$. Denote

$$
M B=\left[\begin{array}{l}
B_{1} \\
B_{2}
\end{array}\right]
$$

Then system in (1) is restricted system equivalent to the following one:

$$
\begin{aligned}
\dot{\tilde{x}}_{1}(t) & =A_{1} \tilde{x}_{1}(t)+B_{1} w(t) \\
0 & =\tilde{x}_{2}(t)+B_{2} w(t)
\end{aligned}
$$

Lemma 2 [5] Let $V$ be a Lyapunov function for system (1)(2). If $\dot{V}+\alpha V-\frac{\alpha}{\bar{w}^{2}} w^{T}(t) w(t) \leq 0$ with $V(x(0))=0$, then $V \leq 1$.

Our main objective is to find a ball

$$
B(0, r)=\left\{x \in \mathbb{R}^{n} \mid\|x\| \leq r\right\}
$$

to bound the reachable set defined as

$$
R_{x} \triangleq\{x(t) \mid w(t) \text { satisfy (1) and (2), } t \geq 0\} .
$$

Theorem 1 If there exist matrices $P>0, Y, H$ and $U$ such that the following LMI holds:

$$
\Lambda=\left[\begin{array}{ccc}
\Lambda_{1} & \Lambda_{2} & Y^{T} B \\
\star & -\operatorname{sym}(H) & H^{T} B \\
\star & \star & -\frac{\alpha}{\bar{w}^{2}} I
\end{array}\right]<0
$$

where

$$
\begin{aligned}
& \Lambda_{1}=\alpha E^{T} P E+\operatorname{sym}\left(Y^{T} A\right) \\
& \Lambda_{2}=\left(P E+E_{0} U\right)^{T}-Y^{T}+A^{T} H
\end{aligned}
$$

and $E_{0}$ is a full column rank matrix satisfying $E^{T} E_{0}=0$. Then the system in (1) is regular, impulse free and the reachable sets of system (1)-(2) is bounded by the ball $B(0, r)$ where

$$
r=\|N\| \sqrt{r_{1}^{2}+r_{2}^{2}}
$$

with

$$
r_{1}=\frac{1}{\sqrt{\lambda_{\min }(\tilde{P})}}, \quad r_{2}=\left\|B_{2}\right\| \bar{w}
$$

with $\tilde{P}=\left(M^{-T} P M^{-1}\right)_{n_{1} \times n_{1}}, M, N$ and $B_{2}$ defined in Lemma 1 .

Proof. Firstly, the regularity and impulse-free characteristics of system (1) are to be established. From the LMI in (6), we can obtain

$$
\begin{array}{r}
\bar{E}^{T} \bar{P}=\bar{P}^{T} \bar{E} \geq 0 \\
\operatorname{sym}\left(\bar{A}^{T} \bar{P}\right)+\bar{Q}<0
\end{array}
$$

where

$$
\begin{aligned}
& \bar{E}=\left[\begin{array}{ll}
E & 0 \\
0 & 0
\end{array}\right], \bar{P}=\left[\begin{array}{cc}
P E+E_{0} U & 0 \\
Y & H
\end{array}\right] \\
& \bar{A}=\left[\begin{array}{cc}
0 & I \\
A & -I
\end{array}\right], \bar{Q}=\left[\begin{array}{cc}
\alpha E^{T} P E & 0 \\
0 & 0
\end{array}\right]
\end{aligned}
$$

It yields from (8) that

$$
\operatorname{sym}\left(\bar{A}^{T} \bar{P}\right)<0
$$

Since $\operatorname{rank}(\bar{E})=\operatorname{rank}(E)=n_{1} \leq n$, there exist nonsingular matrices $\bar{M}$ and $\bar{N}$ such that

$$
\tilde{E}=\bar{M} \bar{E} \bar{N}=\left[\begin{array}{cc}
I_{n_{1}} & 0 \\
0 & 0
\end{array}\right]
$$

Denote

$$
\begin{aligned}
\tilde{A} & =\bar{M} \bar{A} \bar{N}=\left[\begin{array}{ll}
A_{11} & A_{12} \\
A_{21} & A_{22}
\end{array}\right] \\
\tilde{P} & =\bar{M}^{-T} \bar{P} \bar{N}=\left[\begin{array}{ll}
P_{11} & P_{12} \\
P_{21} & P_{22}
\end{array}\right]
\end{aligned}
$$

Considering (7) and the equalities in (10) and (11), we obtain that $P_{12}=0$ and $P_{11}>0$. Then pre-multiplying and post-multiplying (9) by $\bar{N}^{T}$ and $\bar{N}$, respectively, it yields $\operatorname{sym}\left(A_{22}^{T} P_{22}\right)<0$ which implies that $A_{22}$ is nonsingular. Therefore, the pair $(\bar{E}, \bar{A})$ is regular and impulse free. Noting that

$$
\begin{aligned}
\operatorname{det}(s E-A) & =\operatorname{det}(s \bar{E}-\bar{A}) \\
\operatorname{deg}(\operatorname{det}(s E-A)) & =\operatorname{deg}(\operatorname{det}(s \bar{E}-\bar{A}))
\end{aligned}
$$

it is easy to see the system in (1) is regular and impulse free.

Now, we use Lemma 2 to prove that the ball provided in Theorem 1 can bound the reachable set of the system in (1). To this end, we construct the following Lyapunov function:

$$
V(x)=x^{T}(t) E^{T} P E x(t)
$$

Calculating the derivative of $V(x)$, we have

$$
\dot{V}(x)=2 \dot{x}^{T}(t) E^{T}\left(P E+E_{0} U\right) x(t)
$$

Introducing the free weighting matrices $Y$ and $H$, we have

$$
2\left[x^{T}(t) Y^{T}+(E \dot{x}(t))^{T} H^{T}\right][-E \dot{x}(t)+A x(t)+B w(t)]=0
$$

Then, combining (12)-(13) and denoting the augmented system variable as

$$
\xi(t)=\left[\begin{array}{lll}
x^{T}(t) & (E \dot{x}(t))^{T} & w^{T}(t)
\end{array}\right]^{T}
$$

yields

$$
\dot{V}\left(x_{t}\right)+\alpha V\left(x_{t}\right)-\frac{\alpha}{\bar{w}^{2}} w^{T}(t) w(t)=\xi^{T}(t) \Lambda \xi(t)<0
$$

By Lemma 2, we have $x^{T}(t) E^{T} P E x(t) \leq 1$, which implies $\tilde{x}^{T}(t) N^{T} E^{T} M^{T} M^{-T} P M^{-1} M E N \tilde{x}(t) \leq 1$, that is,

$$
\tilde{x}_{1}^{T}(t) \tilde{P} \tilde{x}_{1}(t) \leq 1
$$

with $\tilde{P}=\left(M^{-T} P M^{-1}\right)_{n_{1} \times n_{1}}$. Therefore, the following inequality holds:

$$
\left\|\tilde{x}_{1}(t)\right\| \leq r_{1}
$$

with $r_{1}=\frac{1}{\sqrt{\lambda_{\min }(\tilde{P})}}$.

On the other hand, it follows from (4) that

$$
\left\|\tilde{x}_{2}(t)\right\|=\left\|B_{2} w(t)\right\|
$$

which leads to

$$
\left\|\tilde{x}_{2}(t)\right\| \leq r_{2}
$$

where $r_{2}=\left\|B_{2}\right\| \bar{w}$.

In summary, considering (14) and (15), we obtain that $\|x(t)\|=\|N \tilde{x}(t)\| \leq\|N\| \sqrt{r_{1}^{2}+r_{2}^{2}}$. 
Remark 1 In order to seek the 'smallest' possible radius $r_{1}$, the following additional requirement is added $\delta I \leq$ $\left(M^{-T} P M^{-1}\right)_{n_{1} \times n_{1}}$ which is equivalent to

$$
\begin{gathered}
{\left[\begin{array}{cc}
\bar{\delta} I & I \\
I & \left(M^{-T} P M^{-1}\right)_{n_{1} \times n_{1}}
\end{array}\right]} \\
=\left[\begin{array}{cc}
\bar{\delta} I & I \\
I & \left(M^{-T}\right)_{n_{1} \times n} P\left(M^{-1}\right)_{n \times n_{1}}
\end{array}\right] \geq 0
\end{gathered}
$$

where $\bar{\delta}=\delta^{-1}$ is minimized.

\section{Illustrative Example}

In this section, some examples are provided to illustrate the effectiveness of the proposed approach.

Consider a singular system in (1) with following parameters:

$$
\begin{aligned}
E & =\left[\begin{array}{cc}
-1 & 1 \\
0 & 0
\end{array}\right], A=\left[\begin{array}{ll}
2 & 2 \\
0 & 2
\end{array}\right] \\
B & =\left[\begin{array}{l}
3 \\
1
\end{array}\right], \quad E_{0}=\left[\begin{array}{l}
0 \\
1
\end{array}\right] \\
M & =\left[\begin{array}{cc}
-1 & 2 \\
0 & 1
\end{array}\right], N=\left[\begin{array}{ll}
1 & 0.5 \\
0 & 0.5
\end{array}\right]
\end{aligned}
$$

By solving the LMIs in (6) and (16), the minimized $\bar{\delta}$ is obtained as 0.25 . On the other hand, the system is restricted system equivalent to

$$
\begin{aligned}
\dot{\tilde{x}}_{1}(t) & =-2 \tilde{x}_{1}(t)-w(t) \\
0 & =\tilde{x}_{2}(t)+w(t)
\end{aligned}
$$

Then we have $r_{1}=\sqrt{\bar{\delta}}=0.5$ and $r_{2}=1$. Finally, the radius of the ball is $r=\|N\| \sqrt{r_{1}^{2}+r_{2}^{2}}=1.2792$. Figures $1-3$ are depicted the reachable sets corresponding to different inputs and the bounding circle.

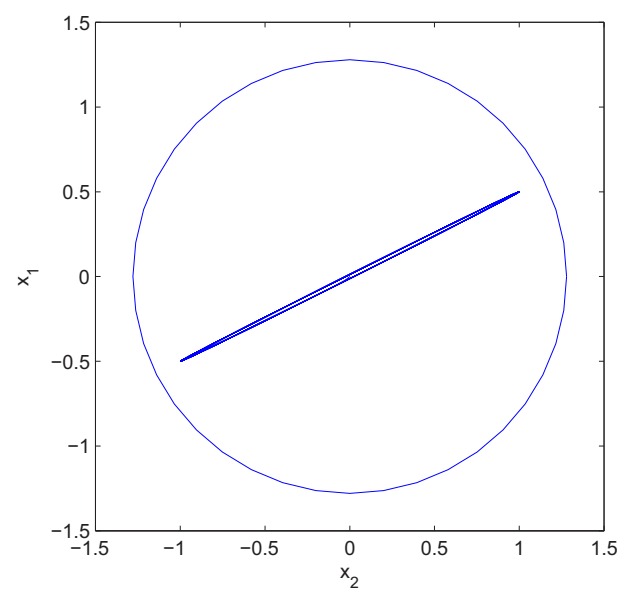

Fig. 1: Reachable set with $w(t)=\sin (0.1 t)$ and the bounding circle

\section{Conclusions}

The problem of reachable set estimation of continuoustime singular systems has been studied for the first time in this paper. A sufficient condition in terms of LMIs has been proposed for guaranteeing the reachable set of singular systems to be bounded by a ball. The results presented in this

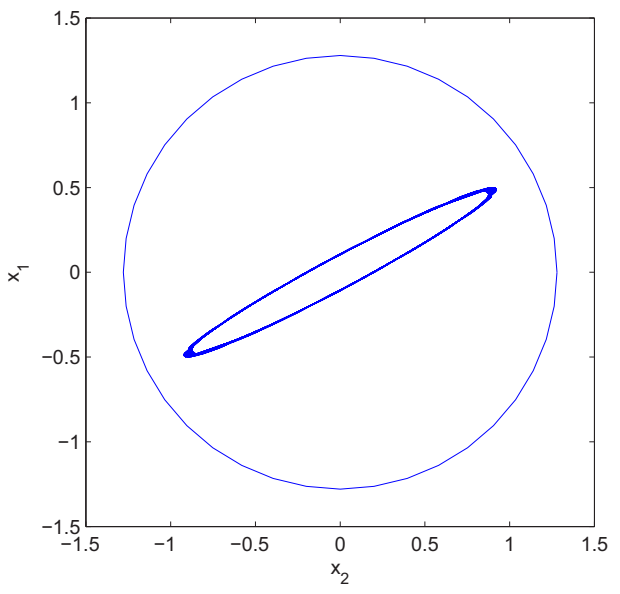

Fig. 2: Reachable set with $w(t)=\sin (t)$ and the bounding circle

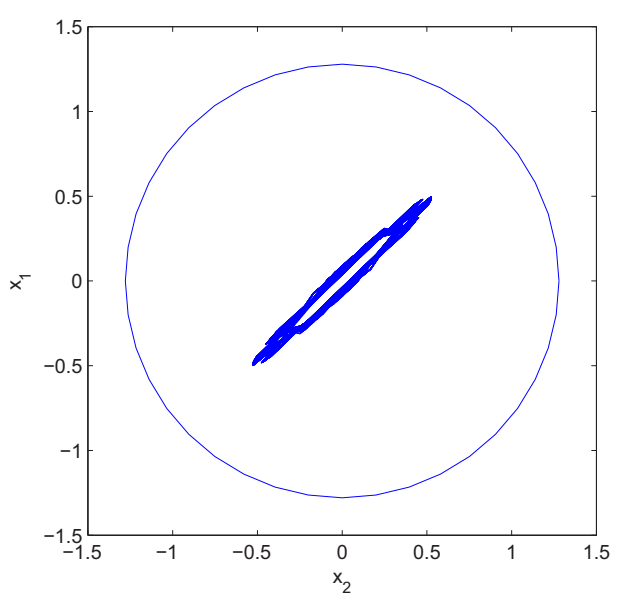

Fig. 3: Reachable set with $w(t)=\sin (10 t)$ and the bounding circle

paper are in terms of strict LMIs which make the conditions more tractable. Finally, numerical examples are given to demonstrate the effectiveness of our methods. On the other hand, the time-delay exists in various practical systems and it is one of the main factors of instability and performance degradation. Therefore, the reachable set estimation problem for singular system with time-delay is an interesting topic for further investigation.

\section{References}

[1] S. Boyd, L. El Ghaoui, E. Feron, and V. Balakrishnan. Linear Matrix Inequalities in System and Control Theory. PA:SIAM, Philadelphia, 1994.

[2] Z. Feng, J. Lam, and H. Gao. $\alpha$-dissipativity analysis of singular time-delay systems. Automatica, 47:2548-2552, 2011.

[3] Z. Feng, J. Lam, H. Gao, and B. Du. Delay-dependent robust $H_{\infty}$ controller synthesis for discrete singular delay systems. Int. J. Robust \& Nonlinear Control, 21:1880-1902, 2011.

[4] E. Fridman. Stability of linear descriptor systems with delay: a Lyapunov-based approach. Journal of Mathematical Analysis and Applications, 273:24-44, 2002.

[5] E. Fridman and U. Shaked. On reachable sets for linear systems with delay and bounded peak inputs. Automatica, 39:20052010, 2003. 
[6] K. Gu, V. L. Kharitonov, and J. Chen. Stability of Time-delay Systems. Birkhaüser, Berlin, 2003.

[7] T. Hu and A. R. Tee and L. Zaccarian Stability and performance for saturated systems via quadratic and nonquadratic Lyapunov functions. IEEE Trans. Automat. Control, 51:17701786, 2006.

[8] J. H. Kim. Improved ellipsoidal bound of reachable sets for time-delayed linear systems with disturbances. Automatica, 44:2940-2943, 2008.

[9] A. Kumar and P. Daoutidis. Feedback control of nonlinear differential-algebraic-equation systems. AIChE Journal, 41:619-636, 1995.

[10] O. Kwon, S. Lee, and J. Park. On the reachable set bounding of uncertain dynamic systems with time-varying delays and disturbances. Information Sciences, 181:3735-3748, 2011.

[11] W. Q. Liu and V. Sreeram. Model reduction of singular systems. Int. J. Systems Sci., 32:1205-1215, 2001.

[12] D. G. Luenberger and A. Arbel. Singular dynamic Leontief systems. Econometrica, 45:991-995, 1977.

[13] P. Nam and P. Pathirana. Further result on reachable set bounding for linear uncertain polytopic systems with interval time-varying delays. Automatica, 47:1838-1841, 2011.

[14] R. Newcomb. The semistate description of nonlinear timevariable circuits. IEEE Trans. Circuits and Systems, 28:62-71, 1981.

[15] P. G. Park, J. W. Ko, and C. Jeong. Reciprocally convex approach to stability of systems with time-varying delays. Automatica, 47:235-238, 2011.

[16] C. Shen and S. Zhong. The ellipsoidal bound of reachable sets for linear neutral systems with disturbances. Journal of the Franklin Institute, 348:2570-2585, 2011.

[17] B. L. Stevens and F. L. Lewis. Aircraft Modeling, Dynamics and Control. New York: Wiley, 1991.

[18] H. S. Wang, C. F. Yung, and F. R. Chang. Bounded real lemma and $H_{\infty}$ control for descriptor systems. IEE Proc. Control Theory Appl., 145:316-322, 1998.

[19] Z. Wu, J. H. Park, H. Shu, and J. Chu. Dissipativity analysis for singular systems with time-varying delays. 218:4605-4613, 2011.

[20] S. Xu and J. Lam. $H_{\infty}$ model reduction for discrete-time singular systems. Systems \& Control Letters, 48:121-133, 2003.

[21] S. Xu and J. Lam. Robust stability and stabilization of discrete singular systems: an equivalent characterization. IEEE Trans. Automat. Control, 49:568-574, 2004.

[22] S. Xu and J. Lam. Control and Filtering of Singular Systems. Springer, Berlin, 2006.

[23] S. Xu, P. Van Dooren, R. Stefan, and J. Lam. Robust stability and stabilization for singular systems with state delay and parameter uncertainty. IEEE Trans. Automat. Control, 47:1122$1128,2002$.

[24] G. Zhang, Y. Xia, and P. Shi. New bounded real lemma for discrete-time singular systems. Automatica, 44:886-890, 2008.

[25] S. Zhu, C. H. Zhang, and J. Feng. Delay-dependent robust stability criterion for two classes of uncertain singular timedelay systems. IEEE Trans. Automat. Control, 52:880-885, 2007.

[26] Z. Zuo, D. W. C. Ho, and Y. Wang. Reachable set bounding for delayed systems with polytopic uncertainties: The maximal Lyapunov-Krasovskii functional approach. Automatica, 46:949-952, 2010. 